\title{
Universality Class of Fluctuating Pulled Fronts
}

\author{
Goutam Tripathy, ${ }^{1}$ Andrea Rocco, ${ }^{2,3}$ Jaume Casademunt, ${ }^{3}$ and Wim van Saarloos ${ }^{1}$ \\ ${ }^{1}$ Instituut-Lorentz, Universiteit Leiden, Postbus 9506, 2300 RA Leiden, The Netherlands \\ ${ }^{2}$ Dipartimento di Fisica, Università di Roma "La Sapienza," Piazzale Aldo Moro 2, I-00185 Roma, Italy \\ and Istituto Nazionale Fisica della Materia, Unità di Roma, Roma, Italy \\ ${ }^{3}$ Departament ECM, Universitat de Barcelona, Avenida Diagonal 647, E-08028 Barcelona, Spain
}

(Received 14 February 2001)

\begin{abstract}
It has recently been proposed that fluctuating "pulled" fronts propagating into an unstable state should not be in the standard Kardar-Parisi-Zhang (KPZ) universality class for rough interface growth. We introduce an effective field equation for this class of problems, and show on the basis of it that noisy pulled fronts in $d+1$ bulk dimensions should be in the universality class of the $((d+1)+1) \mathrm{D}$ KPZ equation rather than of the $(d+1) \mathrm{D} \mathrm{KPZ}$ equation. Our scenario ties together a number of heretofore unexplained observations in the literature, and is supported by previous numerical results.
\end{abstract}

DOI: 10.1103/PhysRevLett.86.5215

Consider spatiotemporal systems in which the important dynamics is governed by the propagation of fronts or interfacial zones separating two domains whose bulk dynamics is relatively trivial or uninteresting. In the presence of fluctuations, the theory of the stochastic behavior of such fronts or interfaces is well developed [1,2]. In particular, it is known that many such fluctuating $d$-dimensional interfaces in $d+1$ bulk dimensions are described by the Kardar-Parisi-Zhang (KPZ) equation [3] for their height $h$,

$$
\frac{\partial h}{\partial t}=\nu \nabla^{2} h+\lambda(\nabla h)^{2}+\eta,
$$

with $\eta$ a random Gaussian noise with correlations

$$
\begin{aligned}
\left\langle\eta\left(\mathbf{r}_{\perp}, t\right)\right\rangle & =0, \\
\left\langle\eta\left(\mathbf{r}_{\perp}, t\right) \eta\left(\mathbf{r}_{\perp}^{\prime}, t^{\prime}\right)\right\rangle & =2 \epsilon \delta^{d}\left(\mathbf{r}_{\perp}-\mathbf{r}_{\perp}^{\prime}\right) \delta\left(t-t^{\prime}\right) .
\end{aligned}
$$

We will follow common practice to refer to this equation as the $d+1$ dimensional $[(d+1) \mathrm{D}] \mathrm{KPZ}$ equation, where the $d$ refers to the dimension of the interface and the +1 to the time dimension; $\mathbf{r}_{\perp}$ denotes the coordinates perpendicular to the direction of propagation of the interface.

The scaling behavior of so many stochastic interfaces falls in the $(d+1) \mathrm{D} \mathrm{KPZ}$ universality class due to the fact that (1) this equation contains all the terms in a gradient expansion which are relevant in a RG sense, and (2) the long wavelength deterministic dynamics of many interfaces is local in space and time, i.e., of the form $v_{n}=$ $v_{n}\left(\nabla h, \nabla^{2} h, \ldots\right)$, expressing that the normal velocity $v_{n}$ becomes essentially a function of the instantaneous slope (angle) and curvature of the interface only. Upon expanding in the gradients, adding noise, and retaining only relevant terms, one then arrives at (1).

The starting point of such an argument, the fact that one can integrate out the internal structure of the interface and on long length and time scales think of it as a mathematically sharp boundary with effective dynamics expressed by a boundary condition $v_{n}=v_{n}\left(\nabla h, \nabla^{2} h, \ldots\right)$ which is local in space and time, is appealing and usu-
PACS numbers: 05.40.-a, 05.70.Ln, 81.10.Aj

ally correct. Intuitively, one associates it with the interfacial zone being sufficiently sharp on a spatial scale. Nevertheless, there have been scattered observations in the literature which indicate that there is more to it: (a) Some continuum reaction-diffusion equations have propagating planar interfaces of finite width which are stable, but which become weakly unstable for discrete particle model equivalents [4], contrary to what the above coarsegraining picture would suggest. (b) The empirical relation observed for the distribution of diffusion limited aggregation (DLA) fingers in a channel and the interface shape of a viscous finger could not be understood from the standard continuum model until the innocuous looking reaction term was regularized [5]; on hindsight, this was because the standard mean-field DLA equations do not give the appropriate "local" boundary conditions of the type $v_{n}=-\mu \nabla_{n} p$. (c) In a simple stochastic particle model with fluctuating fronts, non-KPZ scaling was observed [6] contrary to what one would naively have expected.

It turns out that these observations all have one common denominator $[7,8]$, in that they are related to the existence of two classes of fronts, "pushed" and "pulled" fronts. Pushed fronts are the usual ones: their dynamics is determined by the behavior in the interfacial zone, a region of finite thickness, and their response to the bulk fields is local in space and time $[9,10]$. Pulled fronts, on the other hand, propagate into a linearly unstable state. Although they do not differ noticeably from pushed fronts in their appearance, their dynamics is driven by the growth and spreading of perturbations about the unstable state in the semi-infinite region ahead of the front [9]; hence they are particularly sensitive to slight changes in the dynamics there $[4,11]$. These important differences led two of us [8] to propose recently that fluctuating variants of $d$ dimensional pulled fronts in $d+1$ bulk dimensions would, indeed, not be in the $(d+1) \mathrm{D} \mathrm{KPZ}$ universality class, even though pushed fronts do effectively give local boundary conditions on long length and time scales, and hence $d o$ give rise to $(d+1) \mathrm{D}$ KPZ scaling in the 
absence of coupling to a diffusion or Laplace field in the bulk [12]. Simulations of a simple stochastic lattice model were consistent with these arguments, and with the earlier observations of [6].

In this paper, we will argue that fluctuating pulled fronts are, indeed, in a different universality class from the usual pushed ones which show the standard KPZ behavior. Indeed, we will show that the semi-infinite region ahead of the front cannot be integrated out, and effectively enhances the dimension by 1: we introduce a field equation for fluctuating pulled fronts and argue that $d$-dimensional fronts in $d+1$ bulk dimension are in the universality class of the $((d+1)+1) \mathrm{D}$ KPZ rather than the $(d+1) \mathrm{D} \mathrm{KPZ}$ equation. This surprising scenario, which also builds on the insight of [13] for the stochastic behavior of pulled fronts in one bulk dimension, is fully consistent with our earlier 2D simulations [8] and also with the heretofore unexplained results of [6] in higher dimensions. In addition, as we shall discuss, our scenario leads to a number of interesting new questions and challenges.

A stochastic equation for pulled fronts should obey two requirements: in the usual stochastic lattice models with fronts, no particles are spontaneously generated when there are none already. Second, the average front speed and the local fluctuations ahead of the front remain always finite in such lattice models [14]. The field equation should be consistent with these basic facts. So when we consider a stochastic field equation for $\phi\left(x, \mathbf{r}_{\perp}, t\right)$ in $d+1$ dimensions $\left(x, \mathbf{r}_{\perp}\right)$ of the type

$$
\frac{\partial \phi}{\partial t}=D \nabla^{2} \phi+f(\phi)+g(\phi) \eta,
$$

these requirements put constraints on the function $f$ and the noise term $g(\phi) \eta$. The stochastic noise $\eta\left(x, \mathbf{r}_{\perp}, t\right)$ has delta correlations as in (3), and is interpreted in the Stratonovich sense, but our arguments will not rely on the distinction between Ito and Stratonovich calculus. Stochastic field equations of this type have, e.g., already been used for studying the scaling behavior of homogeneous bulk phases like directed percolation [15]; investigations of noisy fronts in such equations are more recent-see, e.g., [16] for an analysis of stochastic pulled fronts and a discussion of the applicability to various systems. Here we focus on the proper form for an effective stochastic field equation for pulled fronts. For $f(\phi)$, which determines the dynamics of deterministic fronts in the absence of noise, we choose the standard form for pulled front propagation $f(\phi)=\phi-\phi^{3}$, which gives saturation of the field $\phi$ behind the front where $\phi \rightarrow 1$. How should the noise term $g(\phi) \eta$ look [17]? The requirement that if there are no particles $(\phi=0)$ none are created spontaneously implies that there should be no additive noise term, and hence that $g(\phi=0)=0$. For $\phi$ nonzero but small, it is natural to assume a power law behavior $g(\phi) \sim \phi^{\alpha}$; in the studies of the homogeneous bulk properties of directed percolation the choice $\alpha=1 / 2$ was made [15], motivated by the idea that typical bulk fluctuations are of the order of the square root of the particle density. For pulled fronts, however, the dynamically important region is ahead of the front, where $\phi \rightarrow 0$. Our second requirement that the relative fluctuations $g(\phi) \eta / \phi$ remain finite here shows that the natural choice is $\alpha=1$, i.e., $g(\phi) \sim \phi$ for $\phi \ll 1$ [18]. The linearity of $g$ for small $\phi$ is sufficient for our subsequent analysis. In our numerical studies, we have actually taken $g(\phi)=\phi\left(1-\phi^{2}\right)$, a form taken to suppress fluctuations behind the front. This makes it numerically easier to focus on the fluctuations of the front position itself, without affecting the essential results. Specifically, we thus propose as the generic stochastic field equation for pulled fronts

$$
\frac{\partial \phi}{\partial t}=D \nabla^{2} \phi+(1+\eta) \phi\left(1-\phi^{2}\right) .
$$

Let us now turn to the analysis of the stochastic behavior of fronts which propagate along the $x$ direction into the linearly unstable state $\phi=0$. The crucial feature of pulled fronts is that even though the full dynamics of the fronts is nonlinear, it is essentially determined in the "leading edge," the region ahead of the front where $\phi$ remains small enough that the nonlinear saturation term $-\phi^{3}$ which limits the growth plays no role: the linear spreading and growth of perturbations about the state $\phi=0$ almost literally "pull the front along." An important recent development has been the realization that this simple intuitive picture can be turned into a systematic scheme to calculate even the convergence of the front speed to its asymptotic value $v^{*}$. Remarkably, this relaxation is governed by universal power laws which can be calculated exactly even for general equations [9]. The fact that the stochastic fluctuation effects that we want to investigate are dominant relative to the deterministic velocity relaxation terms suggests that we calculate these along similar lines. For the deterministic case $(\eta=0)$, fronts in (5) propagate with an asymptotic speed $v^{*}=2 \sqrt{D}$. In a frame $\xi=x-v^{*} t$ moving with this speed, the asymptotic front solution has an exponential fall-off $\sim e^{-\lambda^{*} \xi}$ with $\lambda^{*}=1 / \sqrt{D}$ for large positive $\xi$. The asymptotic relaxation analysis of deterministic fronts is based on the so-called leading edge transformation $\phi=e^{-\lambda^{*} \xi} \psi$, which transforms (5) into

$$
\frac{\partial \psi}{\partial t}=D \nabla^{2} \psi+\eta \psi-(1+\eta) \psi^{3} e^{-2 \lambda^{*} \xi} .
$$

Here $\psi$ has been written in the frame moving with velocity $v^{*}$ in the $x$ direction: $\psi=\psi\left(\xi, \mathbf{r}_{\perp}, t\right)$.

In the analysis of deterministic fronts [9], the nonlinear term on the right-hand side (which is exponentially small for $\xi \gg 1$ ) essentially plays the role of a boundary condition for the semi-infinite leading edge region where $\phi$ is small - it allows the nonlinear region to match properly to the leading edge which "pulls" the front. As explained above, this holds a fortiori for fluctuating pulled fronts: their stochastic fluctuations are essentially determined by the region where the linearized equation can be used. Now, 
as is well known, upon making a Cole-Hopf transformation $\psi=e^{h}$, the linearized equation transforms to

$$
\frac{\partial h}{\partial t}=D \nabla^{2} h+D(\nabla h)^{2}+\eta
$$

which is nothing but the $((d+1)+1) \mathrm{D} \mathrm{KPZ}$ equation (1) for the $(d+1)$-dimensional field $h\left(\xi, \mathbf{r}_{\perp}, t\right)$.

As illustrated for two bulk dimensions in Fig. 1, the 1D fluctuations in the front position in the propagation direction are defined by tracing a line where $\phi=$ const, e.g., $\phi=1 / 2$. Since $\phi=e^{-\lambda^{*} \xi+h}$, the front fluctuations in the $\xi$ direction are given by $\xi\left(\mathbf{r}_{\perp}, t\right)=h\left(\xi, \mathbf{r}_{\perp}, t\right) / \lambda^{*}+$ $\xi_{0} \approx h\left(\xi_{0}, \mathbf{r}_{\perp}, t\right) / \lambda^{*}+\xi_{0}$, where the constant $\xi_{0}$ is determined by the level curve of $\phi$ which we trace to determine the front position. Thus, indeed, the position fluctuations of a $d$-dimensional pulled front in $d+1$ bulk dimensions map onto the height fluctuations along a line of a KPZ surface in $d+1$ dimensions - see Fig. 1. The growth and roughness exponents are therefore those of the $((d+1)+1) \mathrm{D} \mathrm{KPZ}$ universality class.

The above scenario unifies a number of different results. It can immediately be compared with the simulation results of the stochastic lattice model of [8]. In that paper a 2D lattice model was introduced in which by changing a simple birth and death rule of particles 1D fronts could be tuned from pushed to pulled. The scaling exponents of the pushed model were found to be the standard $(1+1) \mathrm{D}$ $\mathrm{KPZ}$ ones, as it should, while those of the pulled variants were close to those of the $(2+1) \mathrm{D} \mathrm{KPZ}$ universality class. More importantly, without any adjustable parameters, the distribution functions for the long-time saturated width of the fronts in this model for finite transverse width $L_{\perp}$ [19] are completely in accord with our scenario [8].
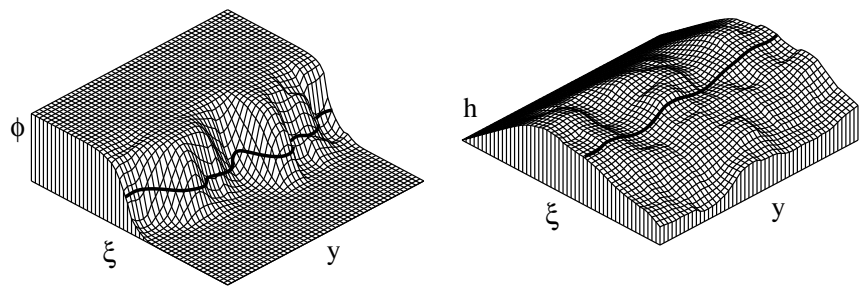

FIG. 1. Left panel: Snapshot of the field $\phi$ at time $t=20$ in a $2 \mathrm{D}$ simulation of (5) with $D=1$ and $\epsilon=10$. The thick line is the position of the front, defined by tracking the line where $\phi\left(\xi, r_{\perp}, t\right)=1 / 2$. Right panel: the same data as in the left figure, plotted in terms of the height variable $h$. Note that $h$ has the appearance of a (slanted) fluctuating surface. The flat portion on the left is the region behind the front and where $h \approx \lambda^{*} \xi$ since $\phi \rightarrow 1$. The thick line indicates the height fluctuations along a line of constant $\xi$. This illustrates that the one-dimensional position fluctuations along the pulled front illustrated by the thick line in the left panel are related to the height fluctuations of the two-dimensional fluctuating surface of the leading edge variable $h$. The scaling behavior of these is that of the $(2+1) \mathrm{D} \mathrm{KPZ}$ universality class.
Moreover, although fronts in 1D do not have transverse fluctuations, the wandering of the position of pulled fronts in one dimension is also consistent with $(1+1) \mathrm{D} \mathrm{KPZ}$ scaling [13]. Finally, the observations of Riordan et al. [6] that in 3 and 4 bulk dimensions their fronts widths did not appear to show a power law growth suggests the following interpretation. According to [8] their fronts are pulled and so they should be governed by the $(3+1) \mathrm{D}$ KPZ equation. The free $\lambda=0$ fixed point in this equation is stable and has no divergent interface width. Apparently above two dimensions the model of $[6,8]$ renormalizes to the weak-coupling fixed point. Probably, by tuning some parameter it can be made to renormalize to the strongcoupling fixed point.

In hindsight, our arguments also justify the regularization of [5] of the mean-field equations for DLA in a channel: the full problem involves pushed fronts, but the mean-field equations have pulled front solutions. The regularization effectively cures this by making the fronts into pushed ones.

The validity of the crucial step of our derivation, the assertion that the nonlinearities in (5) or (6) can be neglected because the leading edge where $\phi \ll 1$ is the essential region, can be tested independently. In Fig. 2 we show simulation data of the wandering of the lines where $\phi=$ 0.5 in Eq. (5) in 2D, both with and without the nonlinearity. Following [21], where the linearized version of (6) was already employed to study the $(2+1) \mathrm{D} \mathrm{KPZ}$ exponents numerically, we have taken parameters so as to make the

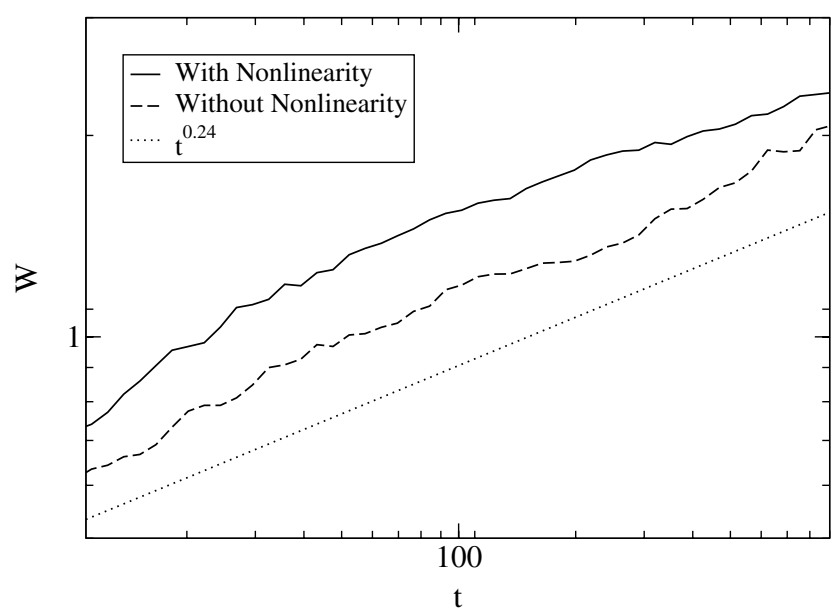

FIG. 2. The increase of the root mean square front width $W=$ $\left[\left\langle\overline{\left[h\left(\mathbf{r}_{\perp}, t\right)-\bar{h}\left(\mathbf{r}_{\perp}, t\right)\right]^{2}}\right\rangle\right]^{1 / 2}$ with time (with the overbar denoting an average over $\mathbf{r}_{\perp}$ ). Data are for simulations of Eq. (6) both with nonlinearity (full line) and without nonlinearity (dashed line), for $\epsilon=5$ and an effective diffusion constant $D=0.4$, which corresponds to dimensionless KPZ coupling constant $\tilde{\lambda}=$ 25 [20]. The front position $h$ in the $x$ direction is defined as the level line where $\phi=0.5$. The fact that the growth exponent is essentially the same with and without $\phi$-nonlinearity justifies our assertion that these terms do not affect the dominant scaling behavior of pulled fronts. 
dimensionless coupling $\tilde{\lambda}=2 \lambda^{2} \epsilon / \nu^{3} \approx 25$. This value appears to be close to the strong coupling fixed point value and so slow transients are minimized [21]. We find, indeed, that the two data sets with and without the nonlinear term in (5) show the same growth exponent, with a value close to the one $\beta \approx 0.24$ of the $(2+1) \mathrm{D} \mathrm{KPZ}$ equation. This gives confidence in the validity of our assertion that the nonlinear terms in the front equation are not important for the scaling behavior of pulled fronts.

The main steps of our line of argument are elegantly direct and build on various previously established ideas; at the same time our scenario also raises a number of new questions and challenges for further research.

(i) There is no systematic theory for the transition from the pushed to the pulled regime in stochastic lattice models, so it is difficult to determine a priori which models lead to the standard pushed fronts and which ones lead to pulled ones. For example, fronts in the directed percolation problem are pushed and obey KPZ scaling in one special case [22], but it is not known whether this is generally so.

(ii) Finite size scaling of the KPZ equation is normally done for interfaces of size $L_{\perp}$ in all directions. Our scenario, on the other hand, leads one to consider anisotropic scaling, since there is effectively a time-dependent cutoff in the $\xi$ direction [13]. The crossover scaling is completely unexplored, but is most likely quite tricky: for fixed $L_{\perp}$ the results of [13] for fronts in one dimension suggest that one should see subdiffusive wandering of the average front position, $\left\langle(\bar{h})^{2}\right\rangle \sim \sqrt{t}$ (rather than $\sim t$ ) because the cutoff in the $\xi$ direction grows as $L_{\xi} \sim \sqrt{t}$, but our simulations seem to suggest that the crossover to this regime happens at such extremely long times that it cannot convincingly be seen in practice. Moreover, the crossover is likely to depend significantly on the initial conditions [13].

(iii) According to the results of $[4,7,11]$, pulled fronts are very sensitive to finite particle effects, so that the convergence to a continuum limit is extremely slow. The results also indicate that any finite particle model has actually weakly pushed fronts, and hence that the true asymptotic regime should be consistent with normal KPZ scaling after all. The anomalous scaling we discussed here then strictly holds only in a field theory without cutoff.

In conclusion, we have put forward an effective field equation for pulled fronts and argued on the basis of it that pulled fronts in $d+1$ bulk dimensions are in the $((d+$ $1)+1) \mathrm{D} K P Z$ universality class rather than the $(d+1) \mathrm{D}$ KPZ universality class. The scenario ties together various results in the literature and brings up various new issues for future research.

W. v. S thanks Uwe Tauber and David Mukamel for stimulating discussions. Financial support from the Dutch science foundation FOM, the Spanish Project No. BXX2000-0638-C02-02, and the TMR network ERBFMRX-CT96-0085 is gratefully acknowledged.
[1] T. J. Halpin-Healy and Y. C. Zhang, Phys. Rep. 254, 215 (1995).

[2] J. Krug, Adv. Phys. 46, 139 (1997).

[3] M. Kardar, G. Parisi, and Y. C. Zhang, Phys. Rev. Lett. 56, 889 (1986).

[4] D. A. Kessler and H. Levine, Nature (London) 394, 556 (1998).

[5] E. Brener, H. Levine, and Y. Tu, Phys. Rev. Lett. 66, 1978 (1991).

[6] J. Riordan, C. R. Doering, and D. ben-Avraham, Phys. Rev. Lett. 75, 565 (1995).

[7] H. Levine, in Proceedings of the 2000 APS March meeting (to be published); L. Pechenik and H. Levine, Phys. Rev. E 59, 3893 (1999).

[8] G. Tripathy and W. van Saarloos, Phys. Rev. Lett. 85, 3556 (2000).

[9] U. Ebert, and W. van Saarloos, Phys. Rev. Lett. 80, 1650 (1998); Physica (Amsterdam) 146D, 1 (2000).

[10] U. Ebert and W. van Saarloos, Phys. Rep. 337, 139 (2000).

[11] E. Brunet and B. Derrida, Phys. Rev. E 56, 2597 (1997).

[12] The Laplacian growth model [2] or DLA in a channel [5] are examples of models with local boundary conditions where the coupling to the Laplacian diffusion field gives non-KPZ behavior.

[13] A. Rocco, U. Ebert, and W. van Saarloos, Phys. Rev. E 62, R13 (2000).

[14] The requirement that the fluctuations in $\phi$ are of order one in the leading edge is in line with the ideas of Brunet and Derrida [11] for the effect of a finite particle cutoff.

[15] H. K. Janssen, Z. Phys. B 42, 151 (1981).

[16] J. Armero, J. Casademunt, L. Ramírez-Piscina, and J. M. Sancho, Phys. Rev. E 58, 5494 (1998).

[17] Actually, as is well known, the Stratonovich field equation is well defined only if the noise correlation length is made finite by writing $\left\langle\eta(\mathbf{r}, t) \eta\left(\mathbf{r}^{\prime}, t^{\prime}\right)\right\rangle=2 \epsilon / \Lambda^{d} C(\mid \mathbf{r}-$ $\left.\mathbf{r}^{\prime} \mid / \Lambda\right) \delta\left(t-t^{\prime}\right)$, with $\int d \mathbf{r} C(\mathbf{r})=1$. This cutoff does not affect the universal long time scaling.

[18] Although our argument is equally valid in the Ito case, we note that in the Stratonovich case, the finiteness of the relative fluctuations and of the renormalized front speed are connected. Indeed, the fluctuation average of the multiplicative noise term is then $\langle g(\phi) \eta\rangle=\epsilon \Lambda^{-d} C(0) \times$ $\left\langle g^{\prime}(\phi) g(\phi)\right\rangle$ (see, e.g., [16]). Here $C(0)$ is the value in the origin of the regularization of the delta function; see [17] above. For $g \sim \phi^{\alpha}$, this gives a term proportional to $\phi^{2 \alpha-1}$, which for $\alpha<1$ leads to an infinite front velocity. Note also that for $\alpha>1$ the analysis in the text implies that the pulled front is unaffected by the noise, so only the choice $\alpha=1$ is consistent.

[19] Z. Rácz and M. Plischke, Phys. Rev. E 50, 3530 (1994).

[20] Our simulations were done on a grid with spacing $\Delta x=$ 1 and bare diffusion constant $D_{0}=0.1$. According to Sec. 5.6.6 of [9] this translates into an effective diffusion coefficient $D=0.40039$ in the $\psi$ equation (6). Our spatial noise correlation function $C$ (see footnote [17]) is a linear function that goes to zero at $\Lambda=5$ grid points.

[21] M. Beccaria and G. Curci, Phys. Rev. E 50, 4560 (1994).

[22] R. Rajesh and D. Dhar, Phys. Rev. Lett. 81, 1646 (1998). 